\title{
Simultaneous Determination of Three Effective Components in Different Compatibility Proportion of the Couple of Sophora flavescens-Fructus Ligustri lucidi by HPLC
}

\author{
Shuhan Chu ${ }^{1}$, Hongxu Zhang ${ }^{1}$, Lixin Ding ${ }^{1} *$ \\ ${ }^{1}$ Key Lab of Biological Medical Preparation, Pharmaceutical School of Jiamusi University, Jiamusi, Heilongjiang, China, \\ 154007
}

\begin{abstract}
Sophora flavescens - Ligustrum lucidum has the effect of increasing the anti-cancer effect of white blood cells and treating liver fibrosis. In order to determine the optimal compatibility ratio of this drug pair, the rationality of the drug pairs in different proportions was revealed. In this experiment, three active ingredients of the Sophora flavescens-Fructus Ligustri lucidi were extracted by reflux extraction, which were matrine, oxymatrine and specnuezhenide. A method for simultaneously determining the content of matrine, oxymatrine and specnuezhenide in a short time by HPLC was established. The method is simple, precise, and stable. Reproducibility can be used to determine the content of three active ingredients of matrine, oxymatrineand specnuezhenide. The analysis results was indicated that the different proportions of compatibility and different extraction solvents have significant effects on the content of active ingredients and dissolution rate of Sophora flavescens- Fructus Ligustri lucidi, after the combination of Sophora flavescens- Fructus Ligustri lucidi in different proportions, the content of matrine, oxymatrine and specnuezhenide is greater than the content of the active ingredients of each single herb. When the ratio of compatibility of Sophora flavescens-Ligustrum Ligustri lucidi is $<1: 1$, it is beneficial to the dissolution of the three active ingredients. By determining the optimal compatibility ratio of this drug pair, provided a theoretical basis for the study of late pharmacodynamics and provide theoretical basis for clinical application of traditional Chinese medicine.
\end{abstract}

\section{Introduction}

The drug pair, is a combination of relatively fixed 2 medicinal tastes commonly used in traditional Chinese medicine, and is a basic form of compatibility of traditional Chinese medicines. Through the combination of the two drugs, synergistic effect or attenuation is played ${ }^{[1]}$. Traditional Chinese medicines will undergo complex chemical composition changes during the compatibility process. In the process of compatibility, attention should be paid to the changes in chemical composition and compatibility pharmacokinetics of each drug. Therefore, this study mainly studied the content of three active ingredients of matrine, oxymatrine and specnuezhenide under the different ratios of sophora flavescens and Fructus Ligustri lucidi, and determined the optimal compatibility ratio.

Sephora flavescens-Fructus Ligustri lucidi was taken from the "Shi Jinmo medicine", The prescription consist of Sephora flavescensa $6 \sim 10 \mathrm{~g}$ and Fructus Ligustri lucidi $10 \sim 15$ g. Sophora flavescens has the disadvantage of immunosuppression; Fructus Ligustri lucidi has the advantage of enhancing immunity. The combination of the two drugs can increase the anticancer effect of white blood cells. Sophora flavescens
Ait, the dry root of Sophora Flavescens Ait, has the effects of dampness, and diuresis. The main active ingredients of Sophora flavescens are matrine and oxymatrine, which have anti-tumor, anti-liver cancer, elevated leukocyte and antibacterial activity. Fructus Ligustri lucidi, the ripe mature fruit of Fructus Ligustri lucidi Ait, is nutritious for liver, kidney, and eyeshades. Specnuezhenide, salidroside, oleanolic acid were the main active ingredients of fructus igustri lucidimain active ingredients for specnuezhenide, salidroside, oleanolic acid, it has the pharmacological effects of protecting liver, anti-cancer and anti-inflammator.

The 2015 edition of the Chinese Pharmacopoeia records that matrine is based on matrine and oxymatrine as quality control standards, and Fructus igustri lucidi is a quality control standard ${ }^{[2]}$. According to the literature, the current research on Fructus igustri lucidi focuses on active components such as oleanolic acid and salidroside ${ }^{[3-4]}$. The chemical composition and pharmacodynamics of the two single-flavored drugs were studied separately. There is no report on the content determination of the different compatibility ratios of Sophora flavescensFructus igustri lucidi ${ }^{[5-7]}$. The HPLC method is used to determine the content of Sophora flavescens in the 2015 edition of the Chinese Pharmacopoeia, the column is an

* Corresponding author: dlx200475@163.com 
$\mathrm{NH}_{2}$ column, most of the literature reports that the content of matrine and oxymatrine is determined by HPLC method using a column as an $\mathrm{NH}_{2}$ column ${ }^{[8-9]}$. Although the HPLC method is used to determine the content of Fructus igustri lucidi, but the column is a C18 column. The detection method of Sophora flavescens and Fructus igustri lucidi in the Chinese Pharmacopoeia cannot be detected at the same time. This study established a HPLC method for the simultaneous determination of three effective components in matrine, oxymatrine and specnuezhenide by HPLC with C18 column. The effects of different proportions of compatibility on the content of these three active ingredients in the drug pair of Sophora flavescensFructus Ligustri lucidi were compared. The dissolution rate of the active ingredient in different extraction solvents is studied, and the optimal extraction solvent of the drug pair is preferred. According to the literature, the optimal ethanol extraction concentration of single-drug is $60 \%-70 \%{ }^{[10-12]}$. Therefore, in this experiment, water and $60 \%$ ethanol were selected as two different extraction solvents to study the effect of the active ingredient on the dissolution rate of the active ingredient of Sophora flavescens and Fructus Ligustri lucidi.

\section{Materials and methods}

\subsection{Chemicals, reagents and materials}

Matrine reference product (batch number: N0622AS, purity $>99.8 \%$ ), oxymatrine reference product (batch number: S0715AS, purity $>99.8 \%$ ), specnuezhenide reference product (batch number: M0621AS, purity $>99.8 \%$ ). They were all purchased from Dalian Meilun Biotechnology Co., Ltd.; acetonitrile and methanol were chromatographically pure, The remaining reagents are of analytical grade, and water was purified.

Model 1100 HPLC (Agilent Corporation, USA), DST100 Traditional Chinese Medicine Crusher (Shanghai dingshuai instrument co. LTD), CP2250 Electronic Analytical Balance (Shanghai orhaus international trading co. LTD), Sophora flavescens and Fructus Ligustri lucidi were purchased from Jiamusi City Jintian pharmacy.

Sophora flavescens and Fructus Ligustri lucidi were purchased from Jintian Pharmacy of Jiamusi City. They were identified by Zong Ximing, senior pharmacist of Pharmacy Experimental Center of Jiamusi University School of Pharmacy, as dry roots of the leguminous plant, and dry fruits of the oleaceae plant.

\subsection{Preparation of mixed reference solution}

The amount of matrine, oxymatrine, and specnuezhenide was accurately placed in a $5 \mathrm{~mL}$ volumetric flask; Methanol was added to volume to obtain a mixed standard solution with concentrations of $50 \mathrm{~g} / \mathrm{mL}, 0.15$ $\mathrm{mg} / \mathrm{mL}$, and $0.25 \mathrm{mg} / \mathrm{mL}$.

\subsection{Preparation of test solution}

According to the ratio of Sophora flavescens to Fructus Ligustri lucidi $(\mathrm{m} / \mathrm{m})$ of $1: 1,1: 2,2: 1,1: 3,3: 1,1: 0$ and $0: 1$, The total amount of the drug pair is $12 \mathrm{~g}$, weigh two copies of each compatibility ratio, were placed in a 250 $\mathrm{ml}$ round bottom flask, the amount of distilled water and $60 \%$ ethanol solution was added 4 times, reflux extraction was Performed twice for $3 \mathrm{~h}$ each time, the extracts were combined and filtered. Methanol were used to concentrate the filtrate and dissolved in a $50 \mathrm{ml}$ volumetric flask, then drawn in a $10.0 \mathrm{ml}$ and $25 \mathrm{ml}$ volumetric flask, adding methanol to a constant volume, where the solution was Penetrate $0.22 \mathrm{~m}$ micro porous membrane and the filtrate was taken, which was the test solution.

\subsection{HPLC system and conditions}

The HPLC system used was an Agilent Technology 1100 series HPLC model equipped with G1311A quaternary pump, G1379A vacuum inline degasser, G1315DAD detector, G1316A column thermostat (USA). And the column was an Agilent 20RBAX XDB-C18 (250 nm $\times$ $4.6 \mathrm{~nm})$. The mobile phase was acetonitrile (A) $-0.2 \%$ aqueous solution of phosphoric acid (B), gradient elution (0 to $15 \mathrm{~min}, 7 \% \mathrm{~A} ; 15$ to $20 \mathrm{~min}, 7 \% \mathrm{~A} \rightarrow 20 \% \mathrm{~A} ; 20$ to $40 \mathrm{~min}, 20 \% \mathrm{~A}$ ) The flow rate was $1.0 \mathrm{~mL} \cdot \mathrm{min}-1$; the detection wavelength was $220 \mathrm{~nm}$; the column temperature was $28^{\circ} \mathrm{C}$; the injection volume was $10 \mathrm{ml}$. Under the above chromatographic conditions, the chromatograms of the mixed standard solution and the test sample are shown in Figure 1.

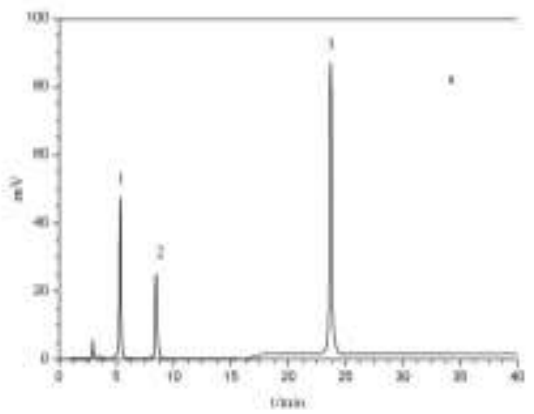

a. Mixed control: 1.matrine; 2. oxymatrine 3. specnuezhenide;

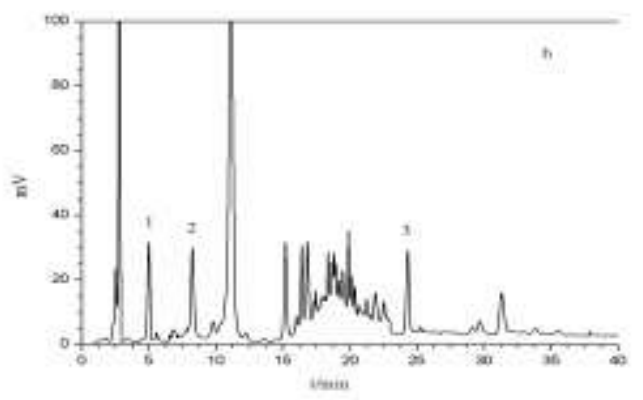

b. Test sample

Fig. 1. HPLC chromatograms of Standards and samples. 


\subsection{Statistical analysis}

Statistical analysis was performed using SPSS 17.0 statistical analysis software. The measurement data was expressed as $\mathrm{X} \pm \mathrm{S}$, using one-way variance. The comparison between groups and analysis of variance was performed. The orderly qualitative data were compared by groups using nonparametric test methods. The difference was statistically significant with $\mathrm{P}<0.05$.

\section{Results and discussion}

\subsection{Optimization of chromatographic conditions}

Mobile phase selection: The effects of methanol-water solution and acetonitrile-water solution on the analysis results were investigated, the analysis results indicated, when methanol-water solution was used as the mobile phase, Retention time of each component was delayed, and the separation Can't satisfied Quantitative requirements. When acetonitrile-aqueous solution was used as the mobile phase, although the peaks of each component can be completely separated, however, there was tailing phenomenon, therefore, the modifier phosphoric acid was added to the aqueous solution. Further examination of the amount of phosphoric acid, by comparison, when acetonitrile- $0.2 \%$ phosphoric acid aqueous solution was used as the mobile phase, the peaks of the component to be measured can be completely separated, the retention time is early, and the shape is better. In order to accurately determine the content of the three active ingredients of matrine, oxymatrine, and specnuezhenide, gradient elution was used.
Detection wavelength selection: Establishment of HPLC method to examine the detection wavelength of each component, the maximum absorption wavelength of matrine and oxymatrine in the 2015 edition of the Chinese Pharmacopoeia is $220 \mathrm{~nm}$. The maximum absorption wavelength of the specnuezhenide was 224 $\mathrm{nm}$. Another report in the literature that specnuezhenide have a greater absorption at wavelengths of $220 \mathrm{~nm}^{[13]}$. Through pre-testing, it was proved that the specnuezhenide area was basically the same at the wavelengths of $220 \mathrm{~nm}$ and $224 \mathrm{~nm}$. In order to detect the contents of three active ingredients of matrine, oxymatrine, and specnuezhenide at the same time more quickly and conveniently, $220 \mathrm{~nm}$ was selected as the detection wavelength in this experiment.

\subsection{Linearity and range}

Precise measurement of the reference solution under "2.2" 1.0, 2.0, 3.0, 4.0, $5.0 \mathrm{ml}$, placed in a $5 \mathrm{~mL}$ volumetric flask. Then methanol was added to volume of a series of mixed reference solutions. Accurately 1.01 of each of the above mixed control solutions was measured to determine the chromatographic conditions under "2.4" and the peak area was recorded. With the mass concentration of the component to be measured $(\mathrm{g} / \mathrm{ml})$ as the abscissa, a standard curve was drawn, the regression equations for matrine, oxymatrine, and specnuezhenide were $\mathrm{y}=12.392 \mathrm{x}-3.7452(\mathrm{r}=0.9971)$, $\mathrm{y}=33.89 \mathrm{x}-3.975 \quad(\mathrm{r}=0.9983), \quad$ and $\quad \mathrm{y}=9.4213 \mathrm{x}-$ $4.3388(\mathrm{r}=0.9994)$.

Table 1. Determination of alcohol extract sample content $(x \pm s, n=3)$

\begin{tabular}{|c|c|c|c|c|c|c|}
\hline A:B & $\begin{array}{c}\text { Matrine } \\
\text { Content } \\
(\mathbf{m g} / \mathbf{g})\end{array}$ & $\begin{array}{c}\text { Matrine } \\
\text { dissolution } \\
\text { rate (\%) }\end{array}$ & $\begin{array}{c}\text { Oxymatrine } \\
\text { content } \\
\mathbf{( m g / g )}\end{array}$ & $\begin{array}{c}\text { Oxymatrine } \\
\text { dissolution } \\
\text { rate (\%) }\end{array}$ & $\begin{array}{c}\text { Specnuezhenide } \\
\text { content } \\
\mathbf{( m g} / \mathbf{g})\end{array}$ & $\begin{array}{c}\text { Specnuezhenide } \\
\text { dissolution } \\
\text { rate (\%) }\end{array}$ \\
\hline $3: 1$ & $0.1017 \pm 0.012$ & 2.259 & $0.1734 \pm 0.021$ & 3.853 & $0.1005 \pm 0.005$ & 6.699 \\
\hline $2: 1$ & $0.0876 \pm 0.007$ & 2.191 & $0.1485 \pm 0.013$ & 3.711 & $0.1405 \pm 0.009$ & 7.027 \\
\hline $1: 1$ & $0.0629 \pm 0.004$ & 2.095 & $0.1066 \pm 0.004$ & 3.553 & $0.2339 \pm 0.001$ & 7.798 \\
\hline $1: 2$ & $0.0400 \pm 0.014$ & 1.999 & $0.0796 \pm 0.023$ & 3.978 & $0.3650 \pm 0.004$ & 9.125 \\
\hline $1: 3$ & $0.0282 \pm 0.009$ & 1.883 & $0.0566 \pm 0.018$ & 3.776 & $0.3959 \pm 0.021$ & 8.798 \\
\hline $1: 0$ & $0.0096 \pm 0.002$ & 0.160 & $0.0309 \pm 0.018$ & 0.515 & 0 & 0 \\
\hline $0: 1$ & 0 & 0 & 0 & 0 & $0.0660 \pm 0.019$ & 1.099 \\
\hline
\end{tabular}

Table 2. Determination of water extract sample content $(x \pm s, n=3)$

\begin{tabular}{|c|c|c|c|c|c|c|}
\hline A:B & $\begin{array}{c}\text { Matrine } \\
\text { Content } \\
\mathbf{( m g / g )}\end{array}$ & $\begin{array}{c}\text { Matrine } \\
\text { dissolution } \\
\text { rate (\%) }\end{array}$ & $\begin{array}{c}\text { Oxymatrine } \\
\text { content } \\
\mathbf{( m g / g )}\end{array}$ & $\begin{array}{c}\text { Oxymatrine } \\
\text { dissolution } \\
\text { rate (\%) }\end{array}$ & $\begin{array}{c}\text { Specnuezhenide } \\
\text { content } \\
\mathbf{( m g / g})\end{array}$ & $\begin{array}{c}\text { Specnuezheni } \\
\text { dedissolution } \\
\text { rate } \mathbf{( \% )}\end{array}$ \\
\hline $3: 1$ & $0.1347 \pm 0.006$ & 2.994 & $0.4927 \pm 0.013$ & 10.95 & $0.1156 \pm 0.009$ & 7.703 \\
\hline $2: 1$ & $0.1134 \pm 0.012$ & 2.834 & $0.4041 \pm 0.011$ & 10.10 & $0.2228 \pm 0.012$ & 11.14 \\
\hline $1: 1$ & $0.0843 \pm 0.019$ & 2.810 & $0.2905 \pm 0.028$ & 9.683 & $0.4154 \pm 0.017$ & 13.85 \\
\hline $1: 2$ & $0.0482 \pm 0.001$ & 2.408 & $0.2514 \pm 0.008$ & 12.57 & $0.5761 \pm 0.013$ & 14.40 \\
\hline $1: 3$ & $0.0387 \pm 0.020$ & 0.861 & $0.1704 \pm 0.012$ & 11.36 & $0.5822 \pm 0.009$ & 12.94 \\
\hline $1: 0$ & $0.0096 \pm 0.017$ & 0.160 & $0.0442 \pm 0.027$ & 0.737 & 0 & 0 \\
\hline $0: 1$ & 0 & 0 & 0 & 0 & $0.0801 \pm 0.025$ & 1.335 \\
\hline
\end{tabular}




\subsection{Precision test}

$1.0 \mathrm{ml}$ of the mixed reference solution under" 2.2 "was taken, and injected 6 consecutive times according to the chromatographic conditions under" 2.4 "to record the peak area. As a result, the RSDs of the peak areas of matrine, oxymatrine, and specnuezhenide were $1.1 \%$, $1.2 \%$, and $1.1 \%(\mathrm{n}=6)$, respectively, and the results showed that the precision of the apparatus was good.

\subsection{Stability test}

The same test substance solution under"2.2"Sophora flavescens-Fructus ligustri lucidi $(1: 3, \mathrm{~m} / \mathrm{m})$ was taken and placed at room temperature for $0,2,4,8,16,24 \mathrm{~h}$, then according to the "2.4" under the chromatographic conditions for measurement, the peak area was recorded. As a result, the RSDs of the peak areas of matrine, oxymatrine, and specnuezhenide respectively were $1.0 \%$, $1.2 \%$ and $1.1 \%(\mathrm{n}=6)$, it shows that the test solution has good stability at room temperature for 24 hours under the conditions of this test.

\subsection{Repeatability test}

Accurately the appropriate amount of Sophora flavescens - Ligustrum lucidum $(1: 3, \mathrm{~m} / \mathrm{m})$ were weighed and test solution was prepared according to the method under "2.3". 6 Parallel copies made to determine the chromatographic conditions under "2.4" and the peak area was recorded. As a result, the RSDs of the peak areas of matrine, oxymatrine, and specnuezhenide were $0.95 \%, 1.2 \%$, and $1.1 \%(\mathrm{n}=6)$, indicating that the method has good reproducibility.

\subsection{Sample recovery test}

An appropriate amount of the known content of Sophora flavescens -Fructus Ligustri licidi $(1: 3, \mathrm{~m} / \mathrm{m})$ was taken. A total of nine and three groups were made. Mixed reference products were added respectively from low, medium and high quality. Prepared the alcohol solution according to the method of "2.3" to provide the sample solution, according to the chromatographic conditions under "2.4", the peak area was recorded. As a result, the range of the recovery rate of the matrine, oxymatrine, and specnuezhenide areas were $95.36 \%, 96.43 \%$ and $95.43 \%$. It shows that the recovery rate of the method is good and feasible.

\subsection{Determination of sample content}

Appropriate amount of Sophorae flavescens - Ligustrum lucidum were taken and the test solution was prepared according to the method under "2.3", then according to "2.4" under the chromatographic conditions for determination, the peak area was recorded, the content and dissolution of the sample was calculated, the results are shown in Table 1 and Table2. A is Sophora flavescens, B is fructus ligustri lucidi.

\subsection{Analysis of results}

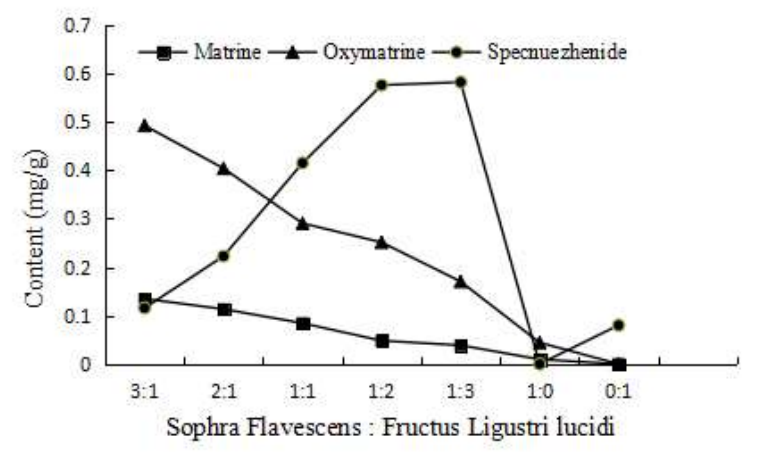

Fig.2. Contents of active constituents in different compatibility ratios of Sophora flavescens and Ligustrum lucidum during ethanol extraction.

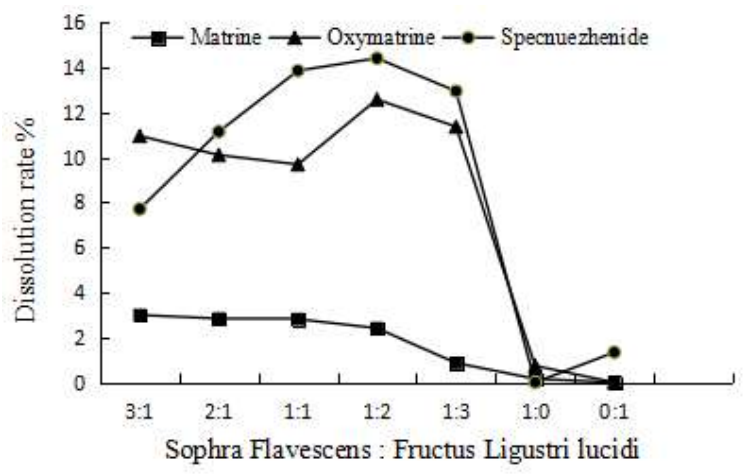

Fig.3. Dissolution rate of active ingredients in different ratios of Sophora flavescens and Ligustrum lucidum during ethanol extraction.

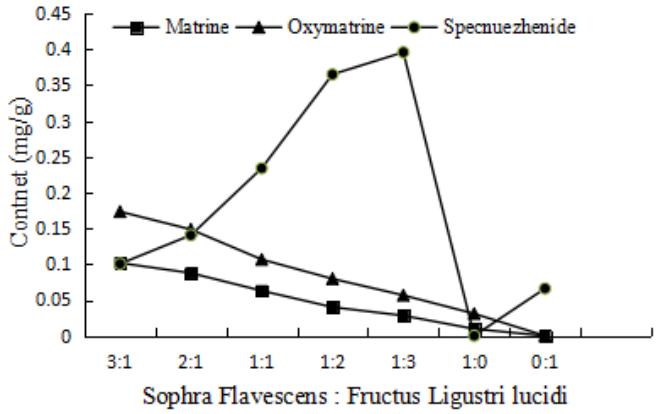

Fig.4. Contents of active constituents in different compatibility ratios of Sophora flavescens and Ligustrum lucidum during water extraction.

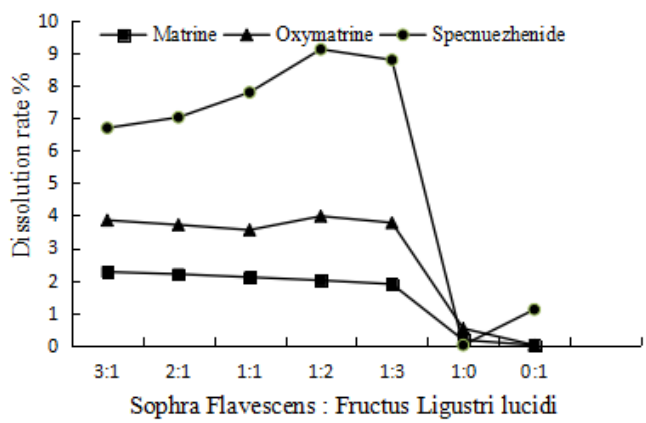


Fig.5. Dissolution rate of active ingredients in different ratios of Sophora flavescens and Ligustrum lucidum during water extraction.

Table 1 shows Figure 2 and Figure 3, Table 2 shows Figure 4 and Figure 5. It can be concluded that the active ingredient in the extraction of Sophora flavescensFructus Ligustri lucidi is superior to the water extraction method by using $60 \%$ ethanol. When water extracting method was used to extract Sophora flavescens-Fructus Ligustri lucidi, had precipitate formation. When $60 \%$ ethanol was used to extracted, the precipitation was reduced. It may be caused by that $60 \%$ ethanol solvent promotes the dissolution of the precipitate and increases the content of the active ingredient. The content and dissolution rate of the active ingredients in the different ratios of Sophora flavescens-Fructus Ligustri lucidi were significantly greater than the active ingredients and dissolution rate of the single-flavored medicines, which increased with the increase of the single-flavored pharmaceutical dosage. The content of matrine and the dissolution rate are not significantly different under different ratios. The effect of the active ingredients oxymatrine and specnuezhenide is significant. When the compatibility ratio is $1: 2$, the dissolution rate of oxymatrine and specnuezhenide is the largest. Xie Ying [14] and other believe that the compatibility is not a simple $1+1$ addition, but in the process of boiling, its chemical properties have undergone qualitative and quantitative changes, thus exerting the unique effects of the compound. Therefore, sophora flavescens and fructus ligustri lucidi may undergo some chemical reaction after compatibility, which promotes the dissolution of matrine, oxymatrine and specnuezhenide, and increases its content. "Shi Jinmo medicine" records 6-10g of Sophora flavescens and 10-15g of Fructus Ligustri lucidi. It can be seen that the dosage of Sophora flavescens is lower than that of Fructus Ligustri lucidi, so the compatibility ratio of $1: 2$ is in line with the scope of the drug. The effects of different compatibility ratios on the content of other ingredients in the Sophora flavescens- Fructus Ligustri lucidi and the pharmacodynamics study need to be further explored to maximize the efficacy.

\section{Conclusions}

In conclusion, HPLC methodestablished in this paper is simple, precise, stable and reproducible. It can be used for the simultaneous determination of three active ingredients in the drug pair of Sophora flavescensFructus Ligustri lucidi, such as matrine, oxymatrine and specnuezhenide.This method is quick and easy. Provided a theoretical basis for the study of late pharmacodynamics. The drug pair of Sophora flavescens-Fructus Ligustri lucidi on different ratios can promote the dissolution of these three active ingredients. The active ingredient in the extraction of Sophora flavescens-Fructus Ligustri lucidi is superior to the water extraction method by using $60 \%$ ethanol. When the ratio of compatibility of Sophora flavescens-Ligustrum Ligustri lucidi is $<1: 1$, it is beneficial to the dissolution of the three active ingredients.
This work was supported by the Jiamusi University President Innovation and Entrepreneurship Fund [grand number XZYF2018-44].

\section{References}

1. Y.P. Tang, X.Y. Shu, W.X. Li, M.Zhu, S.L.Xiu, D.W.Qian, X.S.Fan, J.A.Duan, Drug research (I) the formation and development of drug pairs [J]. Chinese Journal of Traditional Chinese Medicine, 38, 4185-4190 (2013) (in Chinese)

2. National Pharmacopoeia Commission. Pharmacopoeia of the People's Republic of China: one [s]. 2015 edition. Beijing: China Medical Science and Technology Press, 202 (2015) (in Chinese)

3. Y. Wang, Y. Chen, Q.Q.Nie. L.Ling, Y.Zhang, Extraction Process of Oleanolic Acid from Ligustrum lucidum and Its Antioxidant Activity[J]. Jiangsu Agricultural Science, 45, 174-176 (2017) (In Chinese)

4. Z.Y.Li, Q.Li, J.Lü,J.H.Ling, X.H.Yu, X.H.Chen, K.S.Bi, LC-MS determination and pharmacokinetic study of salidroside in rat plasma after oral administration of suspensions of traditional Chinese medicine Erzhi Wan and Fructus Ligustri lucidi[J]. Journal of Pharmaceutical Analysis, 1, 8-12 ( 2011) (In Chinese)

5. H. L.Seo, S.Y.Baek, E.H.Leea, J.H.Lee, S.G.lee,K.Y.Kim, M.H.Jang, M.H.Park, J.H.Kim, K.J.Kim, H.S.Lee, S.C.Ahn, J.R.Lee, S.J.Park, S.C.Kim, Y.W.Kim, Liqustri lucidi Fructus inhibits hepatic injury and functions as an antioxidant by activation of AMP-activated protein kinase in vivo and in vitro[J]. Chemico-Biological Interactions, 262, 57-68 (2017)

6. Q.Li, Y.S.Fan, Z.Q.Gao, K.Fan, Z.J.Liu, Effect of Fructus Ligustri Lucidi on osteoblastic like cell-line MC3T3-E1[J]. Journal of Ethnopharmacology, 170, $88-95$ ( 2015)

7. Y. Zhu, Y.N.Yang, Z.M.Feng, J.S.Jiang, P.C.Zhang, Sophoflavanones A and B, two novel prenylated flavanones from the roots of Sophora flavescens[J]. Bioorganic Chemistry, 79, 122-125 (2018)

8. W.L. Wang, H.X. Zhao, H.Y. Jia, Z.M.Yu, D.H.JuDetermination of matrine and oxymatrine in Sophora flavescens L. from different habitats by HPLC[J]. Chinese Journal of Basic Medicine in Traditional Chinese Medicine, 13, 799-800 (2007) (In Chinese)

9. K. Li, S.D. Wang, L.Q. Wu, H.Tang, Simultaneous determination of matrine and oxymatrine in Radix Sophorae by HPLC [J]. Chinese Journal of Pharmaceutical Sciences, 30, 55-57 (2014) (In Chinese)

10. D. Wang, J. LV, Z.Q. Zhang, L.Zhao, Optimization of extraction process of matrine alkaloids by 
orthogonal test $[\mathrm{J}]$. China Journal of Traditional Chinese Medicine and Information, 18, 51-52(2011) (In Chinese)

11. Q. Zhang, W.J. Shi, X.H. Lian, W.J.Du, A,L,Cao, Optimization of the extraction process of Sophora flavescens [J]. Shanxi Traditional Chinese Medicine, 33, 1408-1409 (2012) (In Chinese)

12. Y.Yang, J.Yi, L.Ma,J.G.Wu, J.Z.Wu, Y.B.Wu, Optimization of extraction process of iridoid glycosides from Ligustrum lucidum by multi-index orthogonal test [J]. Chinese Pharmacy, 27, 39703973. (2016) (In Chinese)
13. J.Y. Zhang, Y. Wang, X.N. Song, Z.Y.Zhang, Simultaneous determination of salidroside, scutellarin and tetraside in Ligustrum lucidum from different producing areas in Guizhou by HPLC [J]. Guizhou Medicine, 41, 1038-1040.(2-17) (In Chinese)

14. Y. Xie, F.Q. Tang, W.J. Xu, H.F.Zhou, J.B.Luo, Effects of different compatibility ratios on the content of active ingredients in ephedra-almonds [J]. Shi Zhen Guo Yi Guo Yao, 23, 2686-2688 (2012) (In Chinese) 